Gut, 1967, 8, 4

\title{
Miliary Crohn's disease
}

\author{
K. W. hEATON, C. F. MCCARTHY, R. E. HORTON, J. S. CORNES, \\ AND A. E. READ
}

From the Departments of Medicine, Surgery and Pathology, Bristol Royal Infirmary

EDITORIAL COMMENT This paper draws attention to an important manifestation of Crohn's disease with miliary peritoneal nodules resembling tuberculosis. It is likely that Crohn's disease may not be considered. The differentiation is essential to enable correct treatment to be given.

It is generally accepted that Crohn's disease (regional enteritis) is an inflammatory process affecting the bowel, in which all layers of the wall are involved. Usually this results in the characteristic 'hose-pipe' appearance, easily recognized at laporotomy, in which the wall of the affected segment is thickened, indurated, and reddened or dusky in colour (Crohn and Yarnis, 1958). Occasionally, on the serosal surface of the intestine, there are seen many tiny pale nodules, resembling the tubercles of miliary tuberculosis. These were noted in some of the earliest descriptions of the disease (Crohn, Ginsburg, and Oppenheimer, 1932; Blackburn, Hadfield, and Hunt, 1939), but they have seldom been commented on in recent discussions of the pathology of regional enteritis (Morson, 1965). When mention has been made of serosal tubercles it has been implied, if not explicitly stated, that they are only found on the surface of obviously diseased bowel (van Patter, Bargen, Dockerty, Feldman, Mayo, and Waugh, 1954; Warren and Sommers, 1954; Pollock, 1958; Avery Jones and Gummer, 1960). Moreover this aspect of Crohn's disease is not referred to at all in standard textbooks of surgery.

The purpose of this paper is to describe three cases in which, at laparotomy, miliary tubercles were the most striking or even the only manifestation of Crohn's disease, with the result that in each case the first diagnosis to be considered was tuberculosis.

\section{CASE REPORTS}

CASE 1 R.W., a schoolboy aged 15, was admitted to hospital in May 1965 with an eight-month history of intermittent diarrhoea. The admission was precipitated by the bursting of an unsuspected ischio-rectal abscess. This was explored and drained and a biopsy was taken from the wall of the abscess. Histology showed noncaseating granulomas containing multinucleate giant cells and no acid-fast bacilli. Crohn's disease was diag- nosed. A barium follow-through appeared to show a short area of stenosis in the terminal ileum but with no dilatation proximally. The perineal wound slowly healed and the boy was sent home. Twenty-four days later he was re-admitted because of persistent abdominal pain, diarrhoea, malaise, and irregular fever up to $100^{\circ} \mathrm{F}$. White cell count was normal apart from a moderate left shift of the neutrophils. A chest radiograph was normal.

At laparotomy, most of the small intestine was found to be covered with white nodules, often coalesced into lines, and having the appearance of tubercles (Figs. 1 and 2). The affected bowel was otherwise normal apart from minimal patchy thickening, and there was no lesion to correspond with the radiological abnormality.

A serosal nodule was removed for histological examination and was found to contain non-caseating granulomas with Langhans giant cells (Fig. 3). No acid-fast bacilli were seen, nor could tubercle bacilli be cultured from a mesenteric lymph node in which the same histological changes were present. Corticosteroid treatment was started on the 21st post-operative day in the form of prednisone $15 \mathrm{mg}$. daily. This was followed by a fall of the temperature to normal and by increased well-being. In a follow-up over 12 months he has remained well and free of gastrointestinal symptoms (see Addendum).

If the histology of the earlier ischio-rectal abscess had not been known, this boy might well have been diagnosed at laparotomy as having early tuberculous peritonitis and have been treated with streptomycin, P.A.S., and I.N.A.H. Instead he was treated with prednisone alone and responded well. A year after his operation he was free of any sign of active Crohn's disease, although the perineal wound had been slow to heal. It is tempting to hope that under the influence of corticosteroids the 'tubercles' have resolved without the disease involving the bowel wall.

CASE 2 S.J., a 53-year-old schoolmistress, was admitted to hospital in October 1959 with a three-month history of mild diarrhoea, weight loss, abdominal discomfort, and tiredness. Physical examination and blood count 

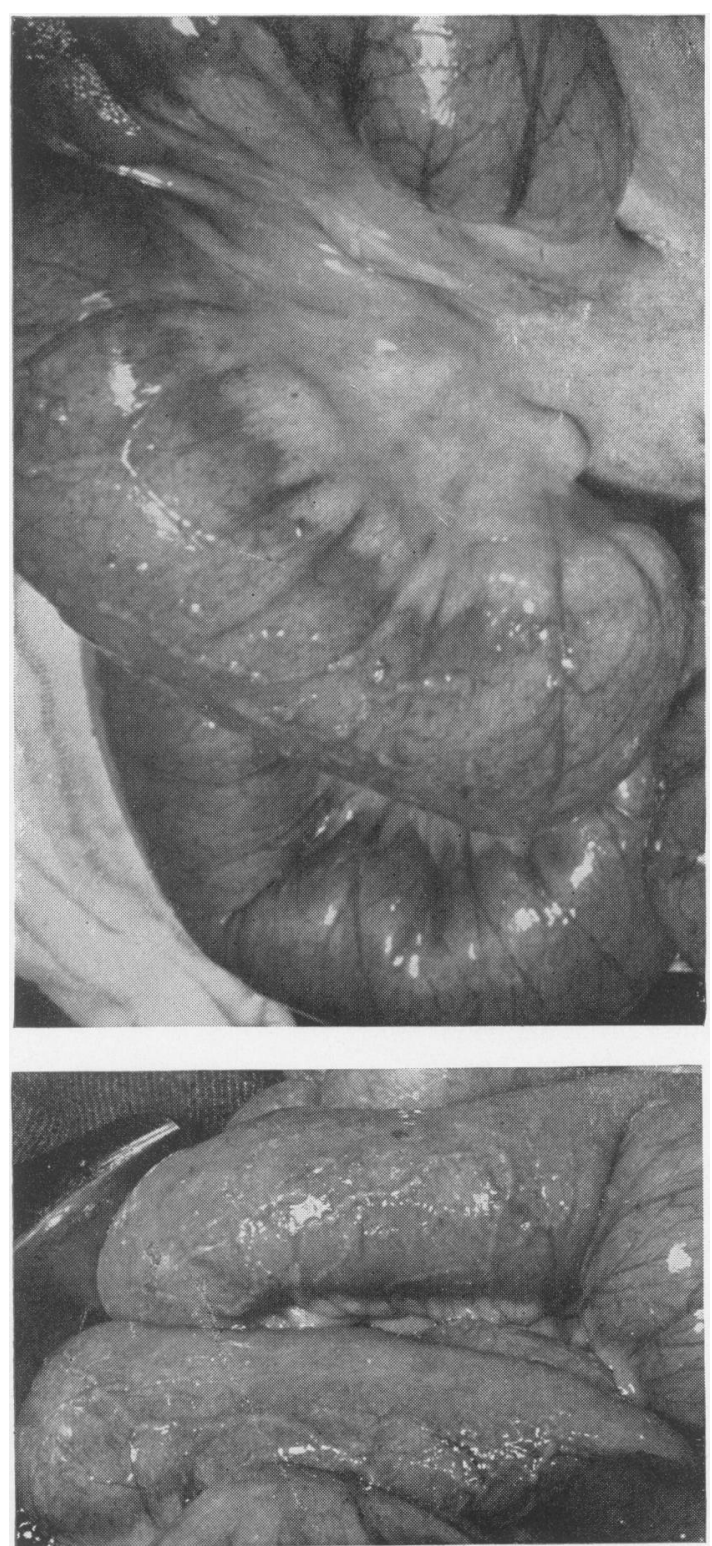

FIGS. 1. and 2. Laparotomy appearances in case 1, showing small intestine covered with miliary 'tubercles' which are tending to coalesce into lines.

were normal. A barium enema was reported as showing a constant narrowing in the upper caecum, highly suspicious of carcinoma, so she was subjected to laparotomy. This revealed some reddening and slight thickening of the terminal ileum but the most striking finding was of numerous 'tubercles' at the ileo-caecal junction. The appendix was white and turgid and the mesenteric lymph nodes were greatly enlarged. The surgeon was so im-

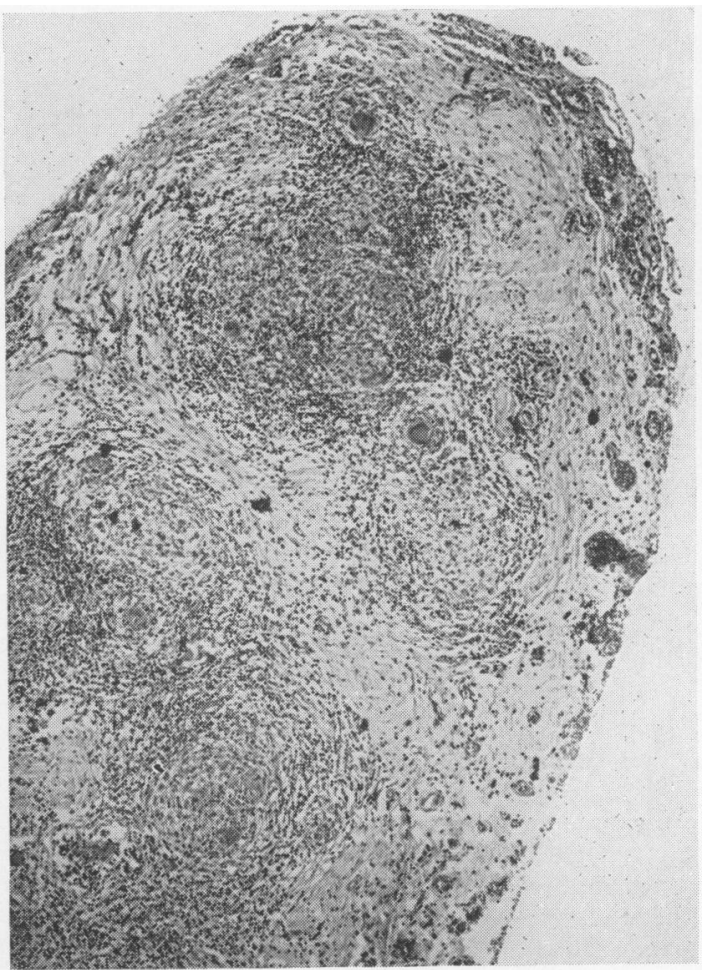

FIG. 3. Histology of a serosal nodule from case 1, showing well-developed granuloma formation with giant cells. (Haematoxylin and eosin $\times 126$.)

pressed by the resemblance to tuberculosis that he ordered streptomycin and I.N.A.H. to be given the same day. Microscopy of the lymph node and appendix showed very numerous non-caseating granulomas with no acidfast bacilli, and a histological diagnosis of sarcoidosis was made. A chest radiograph was normal and a tuberculin skin test was negative using first-strength P.P.D.

Post-operatively anti-tuberculous therapy was continued for 61 days. Thereafter she had only mild gastrointestinal symptoms and received no regular treatment. Eighteen months after the operation a barium followthrough showed the same narrowing in the caecum as before and also some granularity in the appearance of the terminal ileum. It was now recognized that the true diagnosis was Crohn's disease. In subsequent years she has tended to lose weight and to have occasional bouts of abdominal pain, but she has continued working and has not required admission to hospital. Recent $x$-ray studies have shown definite strictures in the terminal ileum, caecum, and ascending colon.

At laparotomy, although there was some evidence of bowel-wall inflammation, the surgeon was so impressed by the 'tubercles' that he diagnosed tuberculosis and ordered immediate anti-tuber- 


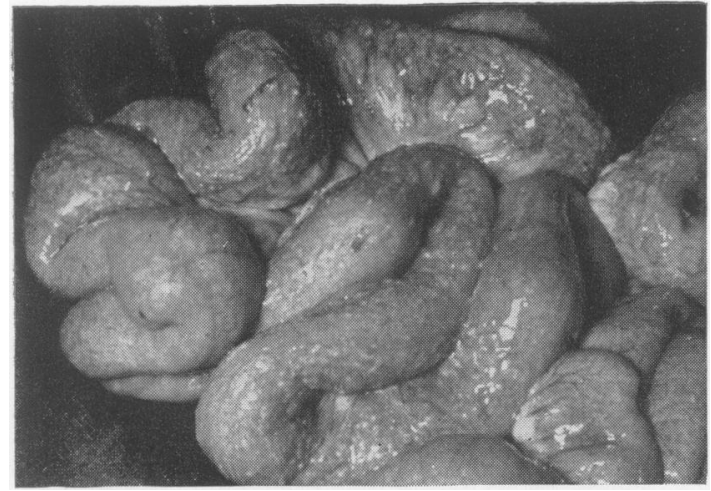

FIG. 4. Laparotomy appearances in case 3, showing extensive involvement of the jejunum with miliary nodules.

culous chemotherapy. This was discontinued after a few weeks. After six years of mild and intermittent symptoms the patient has developed the typical radiological features of Crohn's disease.

CASE 3. B.K., a typist of 16 , was admitted to hospital in February 1960 with an 18-month history of diarrhoea, anorexia, weight loss, and amenorrhoea. These symptoms began two weeks after the death of her father. Examination revealed wasting, a pyrexia up to $99^{\circ} \mathrm{F}$., and a palpable, tender descending colon. Later a mass was palpated in the left iliac fossa. Investigation disclosed a hypochromic anaemia ( $\mathrm{Hb} 78 \%$ ), normal white cell count, E.S.R. $29 \mathrm{~mm}$./hr., normal chest radiograph and normal faecal fat excretion. Barium enema showed extensive narrowing in the descending and sigmoid colon with loss of the mucosal pattern. Barium follow-through showed several areas of irregular narrowing in the small bowel. These radiological appearances received conflicting interpretations. Culture of stool for tubercle bacilli was negative.

At laparotomy there was much free fluid. A $60 \mathrm{~cm}$. section of the upper jejunum was found to be thickened and oedematous and to be covered with numerous white nodules $1-5 \mathrm{~mm}$. in diameter, closely resembling tubercles (Fig. 4). There was also much thickening and oedema in the pelvic colon. A segment of jejunum was resected.

The operative diagnosis was tuberculous enteritis and peritonitis, and treatment with streptomycin and I.N.A.H was started the next day. Histology of the resected jejunum consisted of intense inflammation in all layers with numerous lymphoid follicles and non-caseating granulomas in the subserosa. Post-operative recovery was uneventful. Tuberculin skin testing was negative with both first and second strengths of P.P.D., and after 62 days the anti-tuberculous drugs were discontinued. At this time mild arthritis in the first metacarpo-phalangeal joints was discovered and prednisone therapy was begun. For the next 18 months she kept fairly well but in the following year had three exacerbations requiring admission. In 1964 perforation and abscess formation resulted in resection of $45 \mathrm{~cm}$. of ileum, and in 1965 $37 \mathrm{~cm}$. of diseased colon was removed. At these operations the presence of tubercles was not commented on.
The histology of all specimens has been that of Crohn's disease.

At laparotomy in this case the jejunum and pelvic colon were obviously diseased, but the surgeon was so impressed by the profusion of 'typical tubercles' that he diagnosed tuberculous enteritis and peritonitis and ordered anti-tuberculous chemotherapy. Soon afterwards, when it was apparent that the true diagnosis was Crohn's disease, corticosteroids were substituted and have been continued to the present time. Nevertheless there have been numerous relapses and exacerbations and the patient remains chronically ill.

\section{DISCUSSION}

Tuberculous peritonitis and enteritis are now uncommon diseases in Britain and the United States. Nevertheless, papers have appeared in recent years reporting a continuing and not inconsiderable incidence of tuberculous peritonitis (Burack and Hollister, 1960), tuberculous enteritis (Campbell, 1961; Winter and Goldman, 1966) and ileo-caecal tuberculosis (Campbell, 1961; Howell and Knapton, 1964). It is probable that in the past many cases of Crohn's disease were wrongly diagnosed as tuberculosis, as it is now known that most granulomatous lesions in the ileo-caecal area are due to Crohn's disease (Lee and Roy, 1964). However, even with this knowledge, it may be difficult to distinguish between the two diseases. The situation is complicated by the fact that occasionally nodules closely resembling miliary tubercles are seen on the serosal surface of a segment of bowel affected by Crohn's disease. This fact is not mentioned in the standard textbooks of surgery and is probably not widely appreciated.

That this differential diagnosis may still be difficult is illustrated by the three cases reported here. In two cases the wrong diagnosis was made at laparotomy while in the third case (case 1) the possibility of miliary tuberculosis was considered.

The distinction has some practical importance. While there is no suggestion that anti-tuberculous therapy is harmful (or beneficial) to patients with Crohn's disease, there is good evidence that corticosteroid therapy may be effective in Crohn's disease (Howel Jones and Lennard-Jones, 1966) whereas it may of course be disastrous in tuberculosis.

Study of these three cases yields no single infallible point of distinction between the two diseases, though certain considerations may help in reaching the correct diagnosis when miliary tubercles are found at laparotomy. The presence of ascites may be a point in favour of tuberculosis. It is usual in 
tuberculous peritonitis but uncommon in Crohn's disease (van Patter et al., 1954). The only case of these three in which ascites was encountered at operation was the one with most bowel wall involvement (case 3). The pattern of distribution of the tubercles may be helpful. In miliary tuberculosis the nodules are randomly and evenly scattered while in these cases they tended to coalesce into lines, presumably the lines of the lymphatics. Concentration of the tubercles in the ileo-caecal area should arouse suspicion of Crohn's disease. No help is obtained from white blood cell counts, which were never strikingly abnormal in these cases, nor from the temperature chart, a mild pyrexia being common to both diseases. Chest radiographs were normal in all three patients, but pulmonary lesions are present in only about $50 \%$ of patients with abdominal tuberculosis (Howell and Knapton, 1964). Perhaps the most helpful point in diagnosis is the tuberculin skin test, which was negative in the two patients in this group in whom it was done. It is of course unlikely to be negative in abdominal tuberculosis.

It is of particular interest that in one of our cases (case 1) the bowel itself was macroscopically normal although its serosal surface was extensively covered with miliary tubercles. Perhaps this represents a very early stage in Crohn's disease in which the intestinal wall itself is still healthy. If this is so, the attractive possibility arises of preventing bowel wall involvement by suppressing the diffuse early lesions with corticosteroids. It was with this in mind that case 1 was treated with prednisone. Although his progress to date is very satisfactory he has been followed for only a year. It must be admitted also that case 3 ran a relatively silent course for 18 months before developing a relentless series of exacerbations, even though she remained on corticosteroids throughout. In contrast, case 2 has run a fairly benign course even without corticosteroid treatment. It is perhaps relevant that case 2 most closely resembles case 1 in having had minimal bowel wall involvement at laparotomy. Clearly, however, it is not possible from the available evidence in this small number of patients to make any general statements about the prognosis of this variety of Crohn's disease.

In earlier papers it was stated that when serosal tubercles occur in Crohn's disease they are found histologically to consist of lymphoid collections (van Patter et al., 1954; Pollock, 1958). This is not our experience, for in all three cases the serosal nodules were composed of granulomatous lesions or non-caseating tubercles (Fig. 3).

Little is known about the initial stages in the pathogenesis of Crohn's disease. It has often been suggested that the earliest histological changes result from obstructive lymphoedema, which may in turn be due to lymphadenoid hyperplasia with granuloma formation or to endothelial proliferation in the small lymph vessels (Warren and Sommers, 1948 and 1954). The pathological material in these three cases supports the view that granulomas are the dominant lesions in early subacute Crohn's disease, while the tendency of the serosal nodules to coalesce along the lines of the lymphatics suggests that it is in the lymphatic system that the first pathological changes occur. Moreover, the absence of overt bowel wall involvement in at least one of these cases implies that the peritoneal or mesenteric lymphatic system may be diseased before the bowel itself.

\section{SUMMARY}

Three cases of Crohn's disease are described with predominant involvement of the serosal surface of the intestine. The appearances at laparotomy were suggestive of miliary tuberculosis and led to diagnostic difficulty. The nature and significance of this miliary pattern are briefly discussed.

We wish to thank Dr. John Roylance for his assistance in reviewing the radiographs of the patients.

\section{REFERENCES}

Blackburn, G., Hadfield, G., and Hunt, A. H. (1939). Regional ileitis. St. Bart. Hosp. Rep., 72, 181-224.

Burack, W. R., and Hollister, R. M. (1960). Tuberculous peritonitis. Amer. J. Med., 28, 510-523.

Campbell, E. J. M. (1961). Difficulties in the diagnosis and management of unsuspected tuberculous enteritis and colitis. Gut, 2, 202-209.

Crohn, B. B., Ginsburg, L., and Oppenheimer, G. D. (1932). Regional ileitis; a pathologic and clinical entity. J. Amer. med. Ass., 99, 1323-1329.

- , and Yarnis, H. (1958). Regional Ileitis, 2nd ed. Grune and Stratton, New York and London.

Howel Jones, J., and Lennard-Jones, J. E. (1966). Corticosteroids and corticotrophin in the treatment of Crohn's disease. Gut, 7, $181-187$.

Howell, J. S., and Knapton, P. J. (1964). Ileo-caecal tuberculosis. Ibid. 5, 524-529.

Jones, F. Avery, and Gummer, J. W. P. (1960). Clinical Gastroenterology. Blackwell, Oxford.

Lee, F. D., and Roy, A. D. (1964). Ileo-caecal granulomata. Gut, 5, 517-523.

Morson, B. C. (1965). Crohn's disease of the small intestine. In The Small Intestine, edited by A. C. Thackray and F. Avery Jones, pp. 98-109. Blackwell, Oxford.

Pollock, A. V. (1958). Crohn's disease. Brit. J. Surg., 46, 193-206.

van Patter, W. N., Bargen, J. A., Dockerty, M. B., Feldman, W. H., Mayo, C. W., and Waugh, J. M. (1954). Regional enteritis. Gastroenterology, 26, 347-450.

Warren, S., and Sommers, S. C. (1948). Cicatrizing enteritis (regional ileitis) as a pathologic entity. Amer. J. Path., 24, 475-501. (1954). Pathology of regional ileitis and ulcerative colitis. J. Amer. med. Ass., 154, 189-193.

Winter, J., and Goldman, M, (1966), Tuberculosis of the terminal ileum. Gut, 7 468-480.

\section{ADDENDUM}

Despite continuous treatment with corticosteroid, and the lack of bowel involvement at laparotomy, case 1 has gone on to develop radiological evidence of widespread Crohn's disease of the small and large bowel. He still, however, remains well. 